\title{
Optima and Proxima in Linear Sample Designs
}

\author{
By LESLIE KISH $\dagger$ \\ University of Michigan
}

SUMMARY

The distinct problems of allocating $\sum m_{i}$ sampling units are stated jointly as minimizing $\left(\sum V_{i}^{2} / m_{i}\right)\left(\sum m_{i}\right)$, when either term is fixed, where $\operatorname{var}(\bar{y})=\sum V_{i}^{2} / m_{i}+V_{0}$ and cost $(\bar{y})=\sum c_{i} m_{i}+C_{0}$. The $V_{i}^{2} / m_{i}$ and the $c_{i} m_{i}$ are variance and cost components (strata, stages, phases, etc.); these are affected by the allocation of the $m_{i}$, but the $V_{0}$ and $C_{0}$ are not. The Lagrange identity yields the general relative loss function: $1+L=\left(\sum U_{i} k_{i}\right)\left(\sum U_{i} / k_{i}\right)$, where $L$ is the relative loss, due to relative departures $k_{i} \propto m_{i}^{*} / m_{i}$ from the optimal $L=0$, where the $m_{i}^{*} \propto V_{i} / \sqrt{ } c_{i}$, and the $U_{i}=V_{i} \sqrt{ } c_{i} / \sum V_{i} \sqrt{ } c_{i}$ are relative measures of the components. Solutions are applied to the distinct problems of sample designs. Tables are given of the loss $L$ for useful models of $U_{i}$ and $k_{i}$. The method also leads to useful compromises among the conflicting aims of multipurpose samples, and to measures of relative losses for those aims, e.g. in the conflict between domains and overall means.

Keywords: OPTIMAL ALLOCATION; MULTIPURPOSE ALLOCATION; SAMPLE DESIGNS;

OPTIMAL SAMPLING; EFFICIENT DESIGNS; LINEAR DESIGNS; STRATIFIED ALLOCATION; RELATIVE PRECISION; LAGRANGE MENTITY; DOMAIN ALLOCATION

\section{Basic Definitions and Aims}

IN the literature of survey sampling diverse problems of optimal allocation are treated separately. Yet they can usefully be viewed as distinct examples of the same simple expressions for the total variance and cost of the sample statistic $\bar{y}$ :

$$
\operatorname{var}(\bar{y})=V+V_{0}=\Sigma V_{i}^{2} / m_{i}+V_{0}
$$

and

$$
\operatorname{cost}(\bar{y})=C+C_{0}=\Sigma c_{i} m_{i}+C_{0} .
$$

These linear forms occur in stratified, multistage and multiphase sampling, and other related techniques. Of several applications in Section 7, consider two specific examples. (a) For a stratified sample of elements the variance of the mean $\bar{y}=\Sigma W_{i} \bar{y}_{i}$ is:

$$
\operatorname{var}(\bar{y})=\Sigma\left(W_{i} S_{i}\right)^{2} / m_{i}-\Sigma\left(W_{i} S_{i}\right)^{2} / M_{i},
$$

where $m_{i}, M_{i}, W_{i}$ and $S_{i}^{2}$ are respectively the sample and population sizes, the weights and element variances in the $i$ th stratum. The first term, $V$, depends on the allocation of the $m_{i}$; the second, $V_{0}$ does not. (b) For two-stage random subselection of $b$ from $B$ elements within each of $a$ random selections from $A$ clusters, the variance of the mean is:

$$
\operatorname{var}(\bar{y})=(1-a / A) S_{a}^{2} / a+(1-b / B) S_{b}^{2} / a b=S_{u}^{2} / a+S_{b}^{2} / a b-S_{a}^{2} / A .
$$

Here $S_{u}^{2}=S_{a}^{2}-S_{b}^{2} / B ; V$ comprises the first two terms, with $m_{1}=a$ and $m_{2}=a b$; the last term $-S_{a}^{2} / A=V_{0}$ does not depend on the $m_{i}$. The cost is $c_{a} a+c_{b} a b+C_{0}$.

Definitions and restrictions seem desirable here.

(1) The statistic $\bar{y}$ denotes an estimate of a mean or of an aggregate. Possible extensions to other estimates are not attempted here.

(2) The $i$ th component of the variance, $V_{i}^{2} / m_{i}$, denotes a constant $V_{i}^{2}$ in the design, a unit variance, divided by the number $m_{i}$ of sampling units for that component. We prefer $V_{i}^{2}$ to $V_{i}$ to denote unit variances that are commonly defined with squared values.

$\dagger$ Research Scientist, Institute for Social Research and Professor at the University of Michigan. 
(3) The $i$ th component of cost, $c_{i} m_{i}$, denotes the unit cost $c_{i}$ multiplied by the same number $m_{i}$ of units as in (2).

(4) Components may refer to strata or stages or phases of sampling; generality is the essence of our approach. Components here represent additive sources of variation and cost.

(5) The constants $V_{i}^{2}$ and $c_{i}$ are parameters for which values are assumed or guessed for numerical solutions of allocation problems. We take $V_{i} \geqslant 0$ and $\sqrt{ } c_{i} \geqslant 0$ (hence $V_{i}^{2}$ and $c_{i}$ ) for allocating the $m_{i}$. For non-triviality two pairs at least of the $V_{i}$ and $c_{i}$ should be positive. Negative values of $V_{i}^{2}$ may be encountered, as with $S_{u}^{2}$ above; we then redefine the problem to facilitate a practical solution; for an example see Section 7.3.

(6) The constants $V_{0}$ and $C_{0}$ do not affect optimal allocations of the $m_{i}$; their effects on losses in proximal allocation are shown in Section 3. $C_{0}$ is non-negative in practice, but $V_{0}$ is often negative, as above.

(7) For practical values of $m_{i}$ we want positive integers. Also $0<m_{i} \leqslant M_{i}$, where $M_{i}$ denotes the number of units in the population for the component; and $m_{i} \geqslant 2$ for computing variance components. Frequently, allocation formulae yield some optimal values of $m_{i}^{*}>M_{i}$; when these are reset to $m_{i}=M_{i}$ the other optimal values of $m_{i}^{*}<M_{i}$ can be recomputed with (5.4) and (5.5).

(8) It would seem more realistic to guess distributions for $V_{i}^{2}$ and $c_{i}$, rather than single values, and a Bayesian treatment of design will probably be worth while. But that is beyond our scope here, and I dread a complex procedure out of the reach of survey practitioners. Furthermore, its relative losses would probably not differ much from ours, because losses are insensitive to moderate departures from the guesses.

(9) In some applications, especially for some stratified samples, differences between the $c_{i}$ are disregarded. Hence, the cost constraint becomes $C / c=m=\sum m_{i}$. Then the $\sqrt{ } c_{i}$ should be omitted from the allocation formulae. Instead of $C_{0}$ use $C_{0} / c$, where $c$ is a common (average) unit cost.

(10) This last point calls attention to the dimensional (unit) homogeneity of all the formulae.

To find optimal values $m_{i}^{*} \propto V_{i} / \sqrt{ } c_{i}$ for the $m_{i}$ we minimize the product

$$
V C=\left(\Sigma V_{i}^{2} / m_{i}\right)\left(\Sigma c_{i} m_{i}\right)
$$

when either $V$ or $C$ is fixed at $V_{f}$ or $C_{f}$. This results in the same optimal values as

$$
\operatorname{var}(\bar{y}) \times \operatorname{cost}(\bar{y})=\left(V+V_{0}\right)\left(C+C_{0}\right),
$$

because in $\left(V+V_{0}\right) C_{f}$ or in $V_{f}\left(C+C_{0}\right)$ the second terms are unaffected by optimal allocation; their effects on proxima are more easily treated separately (Section 3 ). To use the product $V C$ rather than some other function seems reasonable: an increase (or decrease) in cost by some factor should be equivalent to a decrease (or increase) in variance by the same factor. The product form leads directly to expressions for loss functions $(1+L)$ and relative losses $(L)$ that are our goals here. For brevity, I use "loss" for $L$ that represents relative increase of variance or cost, without limits.

Our principal aim goes beyond optimization of linear forms, to a simple and coherent treatment of their proximization. We provide convenient forms, in terms of useful parameters, for relative losses incurred by proxima achieved with proximal allocations.

To proximize has the same flavour as Herbert Simon's "to satisfice," but I prefer here the former's neutrality, flexibility and its several forms resembling those of optimize. With these neologisms I want to emphasize a statistical approach, as complementary to the mathematical concept of optimization. Since we cannot attain full optima with designs based on guessed parameters, it is equally important to guess relative losses due to expected sets of departures from optimal allocations. Here we view the optimum as the limit 1 for the loss function $(1+L)$ as $L$ approaches 0 , and how that approach is made. Two designs, one that loses 2 per cent and the other 50, are both non-optimal by strict "optimist" standards; but a 
"proximist" would usually class a 2 per cent loss with the optimal, to distinguish both from larger losses like 50 per cent.

This illustrates that for statisticians "The perfect is the enemy of the good" (proximized from Voltaire). Conflict appears frequently; optimization for one convenient variable often usurps the place of proximization for multipurpose allocation. Proximal methods are seen to be particularly adaptable to multipurpose allocation in Sections 6 and 7.6, and fulfil our second aim.

Further, we also present Section 5, a compact, simple and general formulation of optimal allocations for diverse sampling methods. Instead of solving each separately, we merely substitute appropriate symbols for the optimal values $m_{i}^{*} \propto V_{i} / \sqrt{ } c_{i}$. This is obtained with the simple Cauchy inequality. This unified and simple treatment has heuristic and pedagogic merit. Applications in Section 7 cover the diversity of sampling methods. Sections 2 and 3 develop methods of proximization, and Section 4 contains convenient tables for relative losses $L$.

\section{General Formulation}

Our principal result (2.3) expresses the relative loss $(L)$ in two parameters: $U_{i}$, the relative "sizes" of the components; and $k_{i} \propto m_{i}^{*} / m_{i}$, the relative departures of the sample sizes $m_{i}$ from optimal allocations $m_{i}^{*}$. First $(2.1)$, the product $V C$ to be minimized is divided by $\left(\Sigma V_{i} \sqrt{ } c_{i}\right)^{2}$; this ratio will be shown to have minimal (optimal) value of 1 . It expresses the relative loss $L$ for any allocation of the $m_{i}(>0)$, by compensating for the units of measurement of the $V_{i}^{2}$ and $c_{i}$. Next (2.2), the $m_{i}$ are stated in terms of relative departures $k_{i} \propto m_{i}^{*} / m_{i}$ from their optimal values $m_{i}^{*}$; these will be shown to be $m_{i}^{*} \propto V_{i} / \sqrt{ } c_{i}$. Hence we substitute $m_{i} \propto V_{i} / \sqrt{ }\left(c_{i}\right) k_{i}$ to obtain (2.2); the factors of proportionality cancel. Finally, (2.3) for generality and brevity we substitute the relative "sizes" $U_{i}=V_{i} \sqrt{ } c_{i} / \Sigma V_{i} \sqrt{ } c_{i}$.

$$
\begin{aligned}
1+L & =V C /\left(\Sigma V_{i} \sqrt{ } c_{i}\right)^{2}=\left(\Sigma V_{i}^{2} / m_{i}\right)\left(\Sigma c_{i} m_{i}\right) /\left(\Sigma V_{i} \sqrt{ } c_{i}\right)^{2}, \\
& =\left\{\Sigma V_{i} \sqrt{ }\left(c_{i}\right) k_{i}\right\}\left(\Sigma V_{i} \sqrt{ } c_{i} / k_{i}\right) /\left(\Sigma V_{i} \sqrt{ } c_{i}\right)^{2}, \\
& =\left(\Sigma U_{i} k_{i}\right)\left(\Sigma U_{i} / k_{i}\right),
\end{aligned}
$$

where $U_{i}=V_{i} \sqrt{ } c_{i} / \Sigma V_{i} \sqrt{ } c_{i}$ and $k_{i} \propto V_{i} / \sqrt{ }\left(c_{i}\right) m_{i}$. We take the $k_{i}$ and $U_{i}$ to be positive and finite. We have $\Sigma U_{i}=1$; and we may also use any convenient $U_{i}^{\prime} \propto U_{i}$, if we divide by $\Sigma U_{i}^{\prime}$. Note that we need only the relative values of $k_{i}$, and we can use $A k_{i}$, with $A$ any positive and finite constant. Furthermore, the form of (2.3) shows that the $k_{i}$ may be replaced by their reciprocals; they may refer to ratios of oversampling, as well as undersampling. With this flexibility we can use $\min \left(k_{i}\right)=1$, as we do in Table 1 for convenience.

The minimal value of 1 for (2.3) is obtained with all $k_{i}=k^{*}$ equal. This may appear obvious, or seen with the Lagrange identity in (5.1).

Examples may be useful here.

(a) Consider the variance of the mean $\sum W_{i} \bar{y}_{i}$ for two strata where $W_{1}=0.2, W_{2}=0.8$, $S_{1}^{2}=S_{2}^{2}=S^{2}$ and $c_{1}=c_{2}=c$. Then $U_{i}=W_{i} \propto V_{i}$, and $U_{1}: U_{2}=1: 4$. This implies (5.3) that optimal allocation of sample sizes should be in the ratio of stratum sizes $W_{i}$, hence $m_{2}=4 m_{1}$. If samples of equal sizes, $m_{1}=m_{2}$, are taken, this implies a departure factor of 4 ; we can use simply $k_{1}=1$ and $k_{2}=4$. The consequent relative loss $L$ would be given by $(2.3)$ as $1+L=\left(0.2 \times 1+0.8 \times \frac{1}{4}\right)(0.2 \times 1+0.8 \times 4)=1.360$.

(b) To illustrate the effect of the $U_{i}$ on the loss $L$ : suppose now $S_{1}^{2}=4 S_{2}^{2}$, and $c_{1}=4 c_{2}$. Since $S_{1}^{2} / c_{1}=S_{2}^{2} / c_{2}$, optimal allocation is still $1: 4$. But now $U_{i}=W_{i} S_{i} \sqrt{ } c_{i} / \sum W_{i} S_{i} \sqrt{ } C_{i}$, hence $U_{1}=U_{2}=0.5$. Therefore the relative loss $L$ from equal sample sizes now would be given by $1+L=\left(0.5 \times 1+0.5 \times \frac{1}{4}\right)(0.5 \times 1+0.5 \times 4)=1.5625$.

(c) To illustrate a conflict in allocation: suppose that, as in (a), $S_{1}^{2}=S_{2}^{2}$ and $c_{1}=c_{2}$, but that now we want to minimize the variance of the difference of means $\left(\bar{y}_{1}-\bar{y}_{2}\right)$. Now $U_{1}=U_{2}=0 \cdot 5$. Optimal allocation is at $m_{1}=m_{2}$. Departure from this in the ratio $1: 4$ to satisfy (a) would result in $1+L=(0.5 \times 1+0.5 \times 4)\left(0.5 \times 1+0.5 \times \frac{1}{4}\right)=1.5625$. 
Note that these answers can also be found in Table 1 in column $K=4$ for relative departures. The size of $U$ of one component is 0.2 for (a), and 0.5 for both (b) and (c), in the top two rows. Results for (a) and (c) illustrate common conflicts between totals and domains, treated in Section 7.6 , and in Table 5(A).

The weights $U_{i}$ are convenient for design; based on population parameters, we may call them population weights. However, when dealing with sample results it may be more convenient to use sample weights, based on sample sizes: $u_{i}=U_{i} / k_{i}$. Then (2.3) may be written as

$$
\begin{aligned}
1+L & =\left(\sum u_{i} k_{1}^{2}\right)\left(\sum u_{i}\right), \\
& =1+\left(\sum u_{i} k_{1}^{2} / \Sigma u_{i}-k^{2}\right) / k^{2}=1+C_{k}^{2}, \\
& =1+\sum u_{i}\left(k_{i} / \bar{k}-1\right)^{2} / \Sigma u_{i}, \\
& =1+\sum\left(k_{i} / \bar{k}-1\right)^{2} m_{i} c_{i} / \sum m_{i} c_{i} .
\end{aligned}
$$

$C_{k}^{2}$ is the relative variance (relvariance) of the $k_{i}$ with sample weights $u_{i}$ around their mean $\bar{k}=\sum u_{i} k_{i} / \sum u_{i}=1 / \sum u_{i}$. Here larger $k_{i}>1$ represent larger weights to compensate for undersampling proportionately to their reciprocals. The $u_{i}=U_{i} / k_{i}$ are proportional to $c_{i} m_{i}$ because the $U_{i}=V_{i} \sqrt{ } c_{i} / \Sigma V_{i} \sqrt{ } c_{i} \propto c_{i} m_{i}^{*} \propto c_{i} m_{i} k_{i}$.

\section{On Proximal Allocation}

Extreme departures from optimal values of $m_{i}^{*}$ can result in large relative losses measured in either cost or variance. However, small or even moderate departures from the optimal $m_{i}^{*}$ lead only to negligible or small relative losses. These vague precepts of practising statisticians are given formal and practical expressions (2.4)-(2.7) in terms of the relative loss $(L)$ compared to optimal allocation.

Departures from optimal allocation have several causes. In actual surveys some departures are unavoidable because the true and exact values of $V_{i}^{2}$ and $c_{i}$ are not available. Second, we may further depart from indicated optimal values $m_{i}^{*}$ to convenient proximal integers, or to convenient sampling fractions. Third, surveys generally have several or many purposes, for which the optimal allocations are different. Fourth, the nature of the sampling frame and of the data collection may force departures from optimal selection probabilities; sometimes we are forced to accept unequal selection probabilities when equal probabilities would be close to optimal. Fifth, mistakes in design may be added to the list of good reasons. And sixth, departures from computed values $m_{i}^{*}$ may be forced by the constraints $m_{i} \leqslant M_{i}$ and $m_{i} \geqslant 2$, as noted above.

Thus on many occasions we find it useful to have simple formulations for the relative losses brought about by a set of departures from the optima. We can give useful approximations in terms of the factors $k_{i}$ of relative departures, and of the weights, for different components $i$ of the sample.

When the frequencies for the $k_{i}$ are given or estimated in sample proportions $u_{i}$, then (2.5)-(2.7) yield readily the loss $L$ in terms of the relvariance $C_{k}^{2}$ of the $k_{i}$ values. One of these formulae may be most convenient for judging the losses from actual sample results.

However, for comparing designs of planned samples the frequencies may be more conveniently stated in terms of the population weights $U_{i}$. Formula (2.3) can be readily computed for moderate numbers of components. Furthermore, the simple models of Table 1 can often give instant answers for approximate distributions. I have often found these answers close and adequate for planning designs.

Computations of the relative loss $L$ in our formulae and tables take account of the factors $V$ and $C$ in the minimized function $V C$, but they neglect the constants $V_{0}$ and $C_{0}$ in the total variance and cost (1.1 and 1.2) However, this neglect may be corrected with translations of $L$ into $L^{\prime}$ that does take into account the constant factors $V_{0}$ and $C_{0}$. If $V_{\min }$ is the optimal $V$ 
for fixed $C_{f}$ then the ratio of the attained proximal variance to the optimal variance is

$$
\frac{(l+L) V_{\min }+V_{0}}{V_{\min }+V_{0}}=1+L /\left(1+V_{0} / V_{\min }\right)=1+L^{\prime} .
$$

Thus the adjusted actual relative loss $L^{\prime}$ differs from that indicated by $L$; since $V_{0}$ is often negative, $L^{\prime}$ can be somewhat greater than $L$. For a $C_{\min }$ found for a fixed $V_{f}$, the adjusted relative loss $L^{\prime}$ may be somewhat less than $L$ due to a positive $C_{0}$ in

$$
L^{\prime}=L /\left(1+C_{0} / C_{\min }\right) \text {. }
$$

\section{Tables of Losses for Model Distributions}

For a variety of simple models we can give instant answers about expected losses. Actual population distributions can usually be matched against one of these models so as to provide useful approximations of the expected losses.

The losses are given in terms of departures $k_{i}$ from optimal allocations for the relative weights $U_{i}$ in the models, and the $k_{i}$ range from $\min \left(k_{i}\right)=1$ to $\max \left(k_{i}\right)=K$. The simplest model consists of two components $U$ and $(1-U)$, where the relative departures from optimal sample sizes are in the ratio $k_{1}: k_{2}=1: K$. The loss for two components may be expressed (7.4) as

$$
L=U(1-U)(K-1)^{2} / K .
$$

The dichotomous models represent maximal losses for ranges of departures fixed at 1 to $K$. Thus losses for large values of $K$ are much greater in the top three rows of Table 1 than further down where five other models are shown.

The five models represent diverse frequency distributions for the population weights $U_{i}$; and for each model both discrete and continuous versions are shown. In the discrete versions the relative departures $k_{i}$ take $K$ integral values from 1 to $K$, and the relative weights $U_{i}$ are concentrated at those values. In continuous versions the departures $k_{i}$ and relative weights $U_{i}$ vary continuously from 1 to $K$. Frequencies are divided by their sums to produce relative frequencies $U_{i}$.

Note that the loss $L$ is both very small and uniform for all models for small $K$; for $K=1 \cdot 3$, $L_{d}=0.017$ and $L_{c}=0.006$; for $K=1.5, L_{d}=0.04$ and $L_{c}=0.014$. (Note that for $L_{d}$ the $k_{i}$ take only two values: 1 and $K=1.3$ or 1.5 ). From $K=2$ to about $K=5$ the losses are moderate and fairly similar for the five models. The $L_{c}$ are lower than the $L_{d}$, though in an irregular ratio. Below $K=10$ we can make fairly good guesses about $L$ just from the range 1 to $K$, without knowing much about the $U_{i}$-if this is not dichotomous or $U$-shaped.

However, beyond $K=10$ the losses $L$ increase and diverge. Three of the models show rather similar losses, but for the model $U_{i} \propto 1 / k_{i}$ the losses are much larger. And this model, may often resemble actual frequencies. The fifth has much lower losses, but it is not realistic, I think.

From the models one can also make conjectures about actual distributions that differ somewhat from them. For example, a rectangular distribution for integral values of $k_{i}$ from 1 to 5 has $L_{d}=0.370$; more than five values evenly spaced within the same range to 1 to 5 would have a loss between that value and the continuous loss $L_{c}=0.207$. On the other hand, for only three values of $k_{i}$ from 1 to 5 and $U_{i}=\frac{1}{3}$, the loss (actually 0.533 ) is above 0.370 , but below the dichotomous value of 0.800 in Table 1 .

When sample weights $u_{i}=U_{i} / k_{i}$ seem more convenient, the relative loss $L$ may be estimated by the relvariance $C_{k}^{2}$ of the $k_{i}$, with weights $u_{i}(2.7)$. Formulae and tables can be constructed for such relvariances, if we begin with the means $M$ and variances $\sigma^{2}$ of convenient distributions from 0 to 1 (Kish, 1965, p. 262). To obtain the relvariances $C_{k}^{2}$, those variances are multiplied by the new (range $)^{2}=(K-1)^{2}$ and divided by the new (mean $)^{2}=\{M(K-1)+1\}^{2}$; thus $C_{k}^{2}=\sigma^{2}(K-1)^{2} /\{M(K-1)+1\}^{2}$. Table 3 notes six useful examples. 


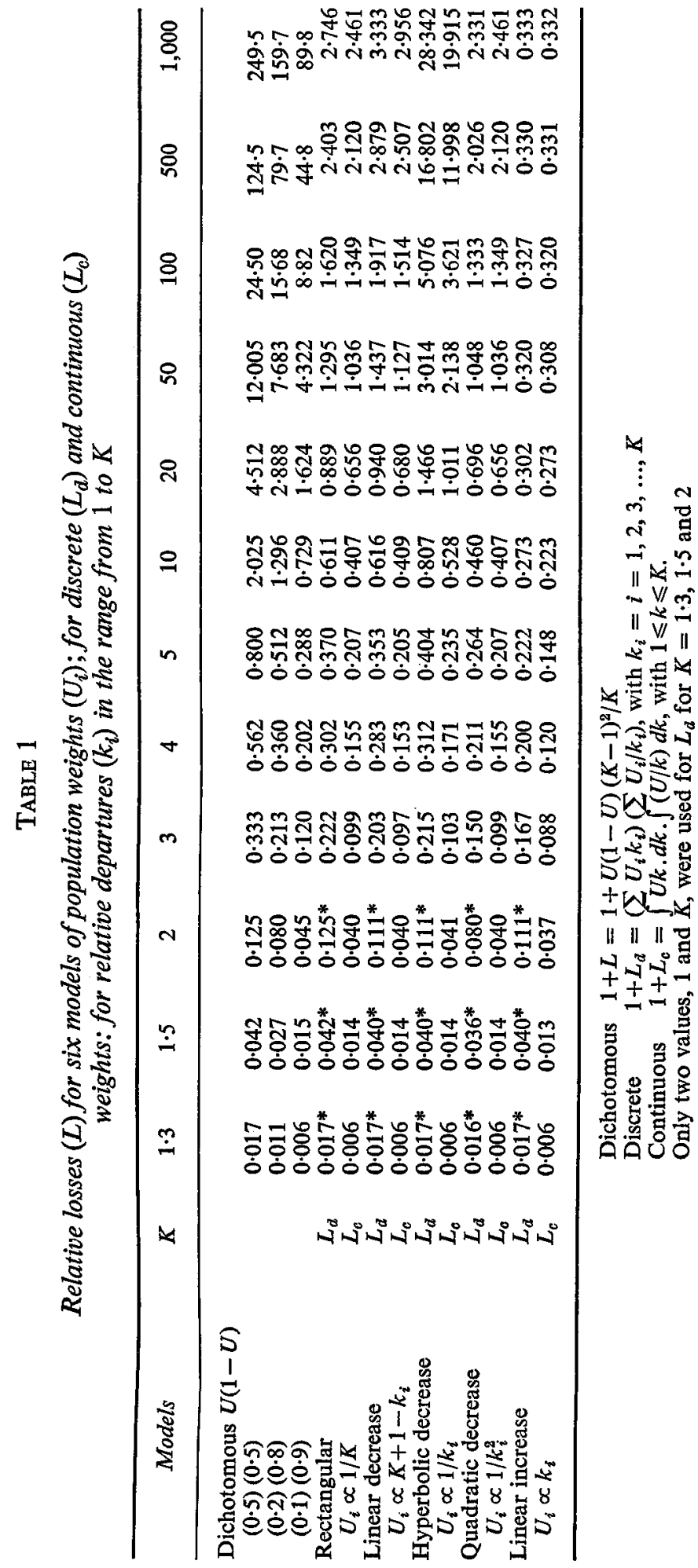




\section{TABLE 2}

Formulae for loss functions $(1+L)$ due to relative departures $k_{i}$ for five models, for both discrete and continuous

\begin{tabular}{lccccc}
\hline Models & $U_{i} \propto 1 / K$ & $U_{i} \propto K+1-k_{i}$ & $U_{i} \propto 1 / k_{i}$ & $U_{i} \propto 1 / k_{i}^{2}$ & $U_{i} \propto k_{i}$ \\
\hline $\begin{array}{c}\text { Discrete } \\
1+L_{a}\end{array}$ & $\frac{K+1}{2 K} \sum \frac{1}{i}$ & $\frac{2(K+2)}{3}\left(\frac{1}{K} \sum \frac{1}{i} \frac{1}{K+1}\right)$ & $\frac{K \sum 1 / i^{2}}{\left(\sum 1 / i\right)^{2}}$ & $\frac{\sum 1 / i \cdot \sum 1 / i^{3}}{\left(\sum 1 / i^{2}\right)^{2}}$ & $1+\frac{K-1}{3(K+1)}$ \\
$\begin{array}{c}\text { Continuous } \\
1+L_{c}\end{array}$ & $\frac{K+1}{2(K-1)} \ln K$ & $\frac{2(K+2)^{2}-3}{3(K+1)(K-1)}\left(\ln K-\frac{K-1}{K+1}\right)$ & $\frac{(K-1)^{2}}{K(\ln K)^{2}}$ & $\frac{K+1}{2(K-1)} \ln K$ & $1+\frac{(K-1)^{2}}{3(K+1)^{2}}$
\end{tabular}

TABLE 3

Losses $L$ for six models of sample weights $u_{i}=U_{i} / k_{i} ;$ the departures $k_{i} \geqslant 1$ from 1 to $K$ represent compensations for undersampling

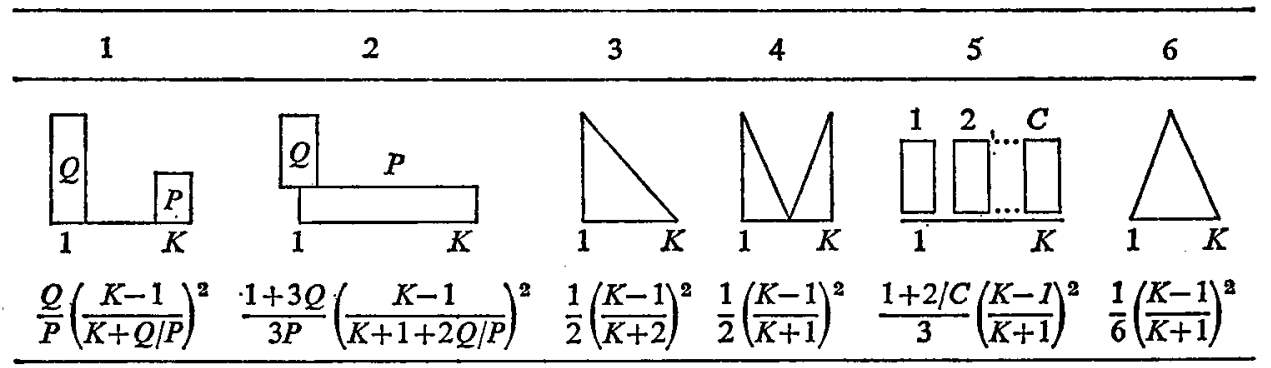

Losses $(L)$ can get large for models 1 and 2 when both $K$ and $Q$ are great: for $K=20$ and $Q=10 P$ the $L$ is 4.011 for 1 and 2.219 for 2 . For the other models losses remain moderate.

\section{On Optimal Allocation}

The Lagrange identity is a basic tool of great utility, and it may be stated here simply. Assume $x_{i}$ and $y_{i}(i=1,2, \ldots, n)$ finite and real; but here we need only non-negative values. Then

$$
\begin{aligned}
\left(\sum x_{i}^{2}\right)\left(\sum y_{i}^{2}\right) & =\sum x_{i}^{2} y_{i}^{2}+\sum_{i \neq j} x_{i}^{2} y_{j}^{2} \\
& =\sum x_{i}^{2} y_{i}^{2}+\sum_{i \neq j} x_{i} y_{i} x_{j} y_{j}+\sum_{i \neq j} x_{i}^{2} y_{j}^{2}-\sum_{i \neq j} x_{i} y_{i} x_{j} y_{j} \\
& =\left(\sum x_{i} y_{i}\right)^{2}+\sum_{i<j}\left(x_{i} y_{j}-x_{j} y_{i}\right)^{2} .
\end{aligned}
$$

The second term has a minimum of 0 , when $y_{i}=F x_{i}, F$ constant. The first term alone is the lower bound of the Cauchy-Schwartz inequality. If we take in (5.1) $x_{i}=\sqrt{(}\left(U_{i} / k_{i}\right)$ and $y_{i}=\sqrt{ }\left(U_{i} k_{i}\right)=k_{i} x_{i}$ we now rewrite (2.3) as

$$
\begin{aligned}
1+L & =\left(\sum U_{i} k_{i}\right)\left(\Sigma U_{i} / k_{i}\right)=\Sigma y_{i}^{2} \sum x_{i}^{2} \\
& =1+\sum_{i<j} \frac{U_{i} U_{j}}{k_{i} k_{j}}\left(k_{i}-k_{j}\right)^{2},
\end{aligned}
$$

with $\left(\Sigma x_{i} y_{i}\right)^{2}=\left(\Sigma U_{i}\right)^{2}=1$. The minimal value is 1 , when the second term is 0 , because all $k_{i}$ are equal. 
Now let $x_{i}=\sqrt{ }\left(V_{i}^{2} / m_{i}\right)$ and $y_{i}=\sqrt{ }\left(c_{i} m_{i}\right)$, with $V_{i}^{2}$ and $c_{i}$ as assumed parameters and $m_{i}$ as variables (all $\geqslant 0)$. The minimal value of

$$
V C=\left(\Sigma V_{i}^{2} / m_{i}\right)\left(\Sigma c_{i} m_{i}\right) \geqslant\left(\sum V_{i} \sqrt{ } c_{i}\right)^{2},
$$

a Cauchy-Schwartz inequality, is obtained when $\sqrt{ }\left(c_{i} m_{i}^{*}\right)=F \sqrt{ }\left(V_{i}^{2} / m_{i}^{*}\right)$. Then

$$
m_{i}^{*}=F V_{i} / \sqrt{ } c_{i}
$$

are the optimal values of the $m_{i}^{*}$ that obtain the

$$
\text { minimal } V C=\left(\Sigma V_{i} \sqrt{ } c_{i}\right)^{2} \text {. }
$$

The constant $F$ can be determined from either $C_{f}$ or $V_{f}$ fixed. With $C_{f}=\sum c_{i} m_{i}^{*}=F \sum V_{i} \sqrt{ } c_{i}$ one uses $F=C_{f} / \sum V_{i} \sqrt{ } c_{i}$. For $V_{f}=\Sigma V_{i}^{2} / m_{i}^{*}$ note that $V_{i} \sqrt{ } c_{i}=F V_{i}^{2} / m_{i}^{*}$ and $\Sigma V_{i} \sqrt{ } c_{i}=F V_{f}$; hence $F=\left(\Sigma V_{i} \sqrt{ } c_{i}\right) / V_{f}$.

Further $V C /\left(\Sigma V_{i} \sqrt{c_{i}}\right)^{2}=1$ yields either $V_{\min }$ for $C_{f}$ or $C_{\min }$ for $V_{f}$. The minimal value of 1 is viewed as the limiting value of $(1+L)$, as the second term approaches zero and as the $m_{i}$ approach the optimal values $m_{i}^{*}$. The relative size of the second term is seen, when divided by the minimal first term $\left(\Sigma V_{i} \sqrt{ } c_{i}\right)^{2}$, as the relative loss $L$, due to proximization.

This formulation of the relative loss is the principal advantage of the Lagrange identity over the Cauchy-Schwartz inequality, which has been used for optimizing stratified samples (Stuart, 1954; Sukhatme, 1970); also over the method of Lagrange multipliers, generally used in sampling literature as $F\left(m_{i}\right)=\Sigma V_{i}^{2} / m_{i}+\lambda\left(\Sigma c_{i} m_{i}-C\right)$, to obtain the same optima. This method, however, can deal with more complex functions, such as $C=c_{0} \sqrt{ } m_{1}+c_{1} m_{1}+c_{2} m_{2}$.

The optimal formulae may give unacceptable answers when high values of $V_{i} / \sqrt{ } c_{i}$ result in $m_{i}^{*}>M_{i}$, the population of units of the $i$ th component. For example, this occurs often in strata with high values of $S_{i}$; also when subsamples from clusters must be confined to the cluster size (Section 7.3). For such components set $m_{i}^{*}=M_{i}$; we may also do this arbitrarily for components when $m_{i}^{*}>M_{i} / 2$, for example. However, changing only those $m_{i}^{*}$ to $m_{i}^{\prime}=M_{i}$ would cause the constraint $V_{f}$ or $C_{f}$ to be missed. To re-establish the constraint we must recompute for the other values of $m_{i}^{*}$ new, higher values $m_{i}^{\prime}$ proportional to them.

Consider the components $V_{i}^{2} / m_{i}$ and $c_{i} m_{i}$ divided into two sets: the complete $(q)$, where $m_{i}^{\prime}=M_{i}$; and the partial $(p)$, where $m_{i}^{\prime}<M_{i}$; denote the sets as $\Sigma_{i}=\Sigma_{q}+\Sigma_{p}$. The complete components $\Sigma_{q}$ are transferred to the constant factors $V_{0}$ and $C_{0}$ : where $m_{i}^{\prime}=M_{i}$ the $m_{i}^{\prime}$ cease to affect the allocation, because components of the variance with the form $V_{i}^{2}\left(1 / m_{i}^{\prime}-1 / M_{i}\right)$ vanish for $m_{i}^{\prime}=M_{i}$. Thus

$$
\operatorname{var}(\bar{y})=\sum_{i} \frac{V_{i}^{2}}{m_{i}^{\prime}}+V_{0}=\sum_{p} \frac{V_{i}^{2}}{m_{i}^{\prime}}+V_{0}+\sum_{q} V_{i}^{2} / M_{i}
$$

and

$$
\operatorname{cost}(\bar{y})=\sum_{i} c_{i} m_{i}^{\prime}+C_{0}=\sum_{p} c_{i} m_{i}^{\prime}+C_{0}+\sum_{q} c_{i} M_{i}
$$

Then we may solve (5.2) and (5.3) with only $p$ components in one of the first terms above and in $\sum V_{i} \sqrt{ } c_{i}$. We compute the residual constant either as $V_{p}=\operatorname{var}(\bar{y})-V_{0}-\Sigma_{q} V_{i}^{2} / M_{i}$, or as $C_{p}=\operatorname{cost}(\bar{y})-C_{0}-\Sigma_{q} c_{i} M_{i}$.

For convenience, since the $m_{i}^{*}$ are already in the right proportions, instead of recomputing (5.2) and (5.3) we may merely increase the $m_{i}^{*}$ proportionately to the new values $m_{i}^{\prime}$ in the subset $\Sigma_{p}$. Thus: for fixed partial cost $C_{p}$, increase the $m_{i}^{*}$ to $m_{i}^{\prime}=m_{i}^{*}\left(C_{p} / \Sigma_{p} c_{i} m_{i}^{*}\right)$, or for fixed partial variance $V_{p}$, increase the $m_{i}^{*}$ to $m_{i}^{\prime}=m_{i}^{*}\left(\Sigma_{p} V_{i}^{2} / m_{i}^{*}\right) / V_{p}$.

Note also that optimal values $V_{\min }$ and $C_{\min }$ obtained with (5.2) without these adjustments should be recomputed to obey the constraints $m_{i} \leqslant M_{i}$. These enforced departures from the optimal $m_{i}^{*}$ will tend to increase the $V$ or $C$ that can be considered as attainable under the restraints. This will affect the relative loss, as $V_{0}$ and $C_{0}$ are treated at the end of Section 3. 


\section{Multipurpose Allocation}

Sample surveys are typically multipurpose in nature, and it seems imperative to extend the methods of allocation to multipurpose designs. For lack of these methods univariate allocation dominates our literature and theory of sampling; practical work is also affected, but less often. The methods for optimization and proximization developed here seem particularly adaptable to multipurpose design. The general form $\sum V_{i}^{2} / m_{i}$ for variances can serve well the many purposes of a sample survey; for the gth purpose the variance will be denoted by $\Sigma_{i} V_{g i}^{2} / m_{i}$.

The many purposes of a single survey may have several sources: (1) A single variable may result in several statistics; e.g. the mean and median of incomes can benefit from different allocations (Kish, 1961). (2) Most surveys obtain results for several variables on a single subject. (3) Furthermore, some surveys are multisubject in character; e.g. with economic, demographic, social variables. (4) Results for subclasses and for their comparisons may be as important as results based on the entire sample. Designs for subclasses often point to different designs and allocations than those for the entire sample. (5) The common but neglected conflict between designs for comparisons between domain means and for the combined mean for the entire sample is developed in Section 7.6.

Suppose a sample is allocated optimally for variate $\ddot{y}^{\prime}$ with $m_{i}^{\prime}$ proportional to $V_{i}^{\prime} / \sqrt{ } c_{i}^{\prime}$, but optimal allocation for another variate $\bar{y}$ would be $m_{i} \propto V_{i} / \sqrt{ } c_{i}$. The loss incurred for $\bar{y}$ can be measured with the departures $k_{i}=m_{i} / m_{i}^{\prime}=\left(V_{i} / V_{i}^{\prime}\right)\left(\sqrt{ } c_{i}^{\prime} / \sqrt{ } c_{i}\right)$ and with weights $U_{i}=V_{i} \sqrt{ } c_{i} / \Sigma V_{i} \sqrt{ } c_{i}$ in formula (2.3). We are mostly concerned with allocation of the $m_{i}$ within one survey sample, so that $\sqrt{ } c_{i}^{\prime} / \sqrt{ } c_{i}=1$. Then the loss function for $\bar{y}$ due to optimization for $\bar{y}^{\prime}$ may be represented by

$$
\begin{aligned}
1+L\left(m_{i}^{\prime}\right) & =\left(\Sigma V_{i}^{2} \sqrt{ } c_{i} / V_{i}^{\prime}\right)\left(\Sigma V_{i}^{\prime} \sqrt{ } c_{i}\right) /\left(\Sigma V_{i} \sqrt{ } c_{i}\right)^{2} \\
& =\Sigma\left(\frac{V_{i} \sqrt{ } c_{i}}{\sum V_{i} \sqrt{ } c_{i}}\right)^{2} /\left(\frac{V_{i}^{\prime} \sqrt{ } c_{i}}{\Sigma V_{i}^{\prime} \sqrt{ } c_{i}}\right)
\end{aligned}
$$

This may be regarded as the relvariance of $k_{i}=V_{i} / V_{i}^{\prime}$ with weights $u_{i}=V_{i}^{\prime} \sqrt{ } c_{i}(2.7)$. Often the cost factors are constant or disregarded, and (6.1) has a particularly simple form

$$
1+L\left(m_{i}^{\prime}\right)=\Sigma\left(V_{i} / \Sigma V_{i}\right)^{2} /\left(V_{i}^{\prime} / \Sigma V_{i}^{\prime}\right)
$$

If the $m_{i}$ allocated for one survey with $c_{i}$ are used for another with $c_{i} \neq c_{i}^{\prime}$, then we rewrite (6.1), with $V_{i}^{\prime} \sqrt{ } c_{i} / \sqrt{ } c_{i}^{\prime}$ in place of $V_{i}^{\prime}$, as

$$
1+L\left(m_{i}^{\prime}\right)=\left(\Sigma V_{i}^{2} \sqrt{ } c_{i}^{\prime} / V_{i}^{\prime}\right)\left(\Sigma V_{i}^{\prime} \sqrt{ } c_{i} / \sqrt{ } c_{i}^{\prime}\right) /\left(\Sigma V_{i} \sqrt{ } c_{i}\right)^{2} .
$$

Now consider a loss function for several variates indexed with $(g=1,2,3, \ldots)$. The loss function, for a fixed cost $C_{f}=\Sigma c_{i} m_{i}$, may be expressed for each as $1+L_{g}=\left(\Sigma V_{g i}^{2} / m_{i}\right) / V_{g \min }$, where the denominator denotes the minimal variance attainable and computed for the $g$ th variate. Assign the weights $I_{g}\left(\sum I_{g}=1\right)$ to denote the relative importance of the lost precision of the $g$ th variate. Then consider the total expected loss as a linear function of the quadratic loss functions (for a fixed set of $m_{i}$ ) of the variances

$$
\begin{aligned}
1+L\left(m_{i}\right) & =\sum_{g} I_{g}\left(1+L_{g}\right)=1+\sum_{g} I_{g} L_{g}\left(m_{i}\right)=\sum_{g} I_{g}\left(\left(\sum_{i} V_{g i}^{2} / m_{i}\right) / V_{g \min }\right\} \\
& =\sum_{i} \frac{1}{m_{i}} \sum_{g} \frac{I_{g} V_{g i}^{2}}{V_{g \min }}=\sum_{i} \frac{Z_{i}^{2}}{m_{i}},
\end{aligned}
$$

where $Z_{i}^{2}=\Sigma_{g} I_{g} V_{g i}^{2} / V_{g \min }$. Changing the order of summation permits defining this $i$ th component that can be computed. For the multipurpose joint allocation we may compute 
(5.3) the

$$
\text { optimal } m_{i}^{* *}=\frac{Z_{i}}{\sqrt{c_{i}}} \frac{C_{f}}{\sum Z_{i} \sqrt{c_{i}}}
$$

and

$$
1+L\left(m_{i}^{* *}\right)=V_{\min }=\left(\Sigma Z_{i} \sqrt{ } c_{i}\right)^{2} / C_{f} .
$$

From the multipurpose optimal allocations $m_{i}^{* *}$ we may compute the loss function $1+L_{g}\left(m_{i}^{* *}\right)$ for the $g$ th variate considered separately. For each of these we can use (6.1) with $V_{i}=V_{g i}, V_{i}^{\prime}=Z_{i}, k_{g i}=V_{g i} / Z_{i}$ and $U_{g i}=V_{g i} \sqrt{ } c_{i} / \Sigma V_{g i} \sqrt{ } c_{i}$. These may be averaged with the weights $I_{g}$ to obtain the joint loss function (6.3) of $1+L\left(m_{i}^{* *}\right)$ with the multipurpose optimal allocations $m_{i}^{* *}$.

This however may be obtained more directly from (6.4) or (6.5). Thus

$$
\begin{aligned}
1+L\left(m_{i}^{* *}\right) & =\sum_{i} \frac{Z_{i}^{2}}{m_{i}^{* *}}=\left(\sum_{i} Z_{i} \sqrt{ } c_{i}\right)^{2} / C_{f} \\
& =\frac{1}{C_{f}}\left\{\sum_{i} \sqrt{ } /\left(\sum_{g} \frac{I_{g} V_{g i}^{2} c_{i}}{V_{g \min }}\right)\right\}^{2} .
\end{aligned}
$$

When we accept (from (5.2) $V_{g \min }=\left(\sum V_{g i} \sqrt{ } c_{i}\right)^{2} / C_{f}$, we obtain a simpler form, because $V_{g i}^{2} c_{i} / V_{g \min }=\left(V_{g i} \sqrt{ } c_{i} / \Sigma V_{g i} \sqrt{ } c_{i}\right)^{2}$. Thus the jointly determined minimal loss function becomes

$$
\begin{aligned}
1+L\left(m_{i}^{* *}\right) & =\left[\sum_{i} \sqrt{ } /\left\{\sum_{g} I_{g}\left(V_{g i} \sqrt{ } c_{i} / \sum_{i} V_{g i} \sqrt{ } c_{i}\right)^{2}\right\}\right]^{2} \\
& =\left\{\sum_{i} \sqrt{ }\left(\sum_{g} I_{g} U_{g i}^{2}\right)\right\}^{2} .
\end{aligned}
$$

The minimal and optimal values may be unobtainable, due chiefly to the constraints $m_{i}^{*} \leqslant M_{i}$ (Section 5). In that case the above loss function overestimates the losses incurred over obtainable values of $V_{g \min }$. Note also that using these leads to $Z_{i} \sqrt{ } c_{i}=\sqrt{ }\left(\Sigma_{g} I_{g} U_{g i}^{2}\right) \sqrt{ } C_{f}$, hence to

$$
\text { optimal }\left(m_{i}^{* *}\right)=\frac{\sqrt{ }\left(\sum I_{g} U_{g i}^{2}\right)}{\sum \sqrt{ }\left(\sum I_{g} U_{g i}^{2}\right)} \frac{C_{f}}{c_{i}} .
$$

These can be seen applied in Section 7.6 to the important and frequent conflict between allocations for weighted totals and for comparisons of domains. Two examples are shown in Table 7.5. Note in the last column of Table 5(B) how encouragingly insensitive are the values of (6.7) for moderate differences in the assignments of $I_{g}$.

The weighted mean of relative quadratic losses (6.3) is a modified version of a function proposed by Dalenius (1957, Chapter 9). Another version (Yates, 1960; Cochran, 1963) uses $\sum_{g} I_{g}^{\prime} \sum_{i} V_{g i}^{2} / m_{i}$ the weighted average of variances. Our (6.3) can be easily adapted by using $T_{i}^{2}=\Sigma_{g} I_{g}^{\prime} V_{g i}^{2}$ instead of $Z_{i}^{2}$; in this formulation the weights $I_{g}^{\prime}=I_{g} / V_{g m i n}$ include the minimal variances. This may appear simpler, but it is less explicit.

The optimal allocation of $m_{i}^{*} \propto Z_{i} / \sqrt{ } c_{i}$ can also be obtained with Lagrange multipliers applied to the function

$$
F\left(m_{i}\right)=\sum_{g} I_{g} \sum_{i} V_{g i}^{2} / m_{i} V_{g \min }+\lambda \Sigma c_{i} m_{i}
$$

With Lagrange multipliers we also investigated two other loss functions: the product, $\Pi\left(1+L_{g}\right)$, and the sum of the relative precisions, $\left\{\Sigma\left(1+L_{g}\right)^{-1}\right\}^{-1}$. But the results seem less crucial than good choices for the weights $I_{g}$ of relative importance. 
Our methods here aim to minimize the first term of $V+V_{0}$ for fixed $C_{f}$. In situations where $V_{0}$ is considerable, the actual loss should be modified to $L^{\prime}=L /\left(1+V_{0} / V_{\mathrm{min}}\right)$, as noted in Section 3. Furthermore, I consider fixing $C_{f}$ more practical than trying to fix values for a set of $V_{g}$ and then to minimize $C_{f}$. This problem seems to have been solved with "convex programming" on several separate occasions (Srikantan, 1963, and Hartley, 1965, for example), but I do not find this approach useful.

\section{SeVERAl Applications}

\subsection{Stratified Element Sampling}

In this common application the variance and cost functions can be written, with $\Sigma W_{i}=1$, as $V+V_{0}=\Sigma\left(W_{i} S_{i}\right)^{2} / m_{i}-\Sigma\left(W_{i} S_{i}\right)^{2} / M_{i}$ and $C+C_{0}=\Sigma c_{i} m_{i}+C_{0}$. Here $V_{i}=W_{i} S_{i}$ and $U_{i}=W_{i} S_{i} \sqrt{ } c_{i} / \Sigma W_{i} S_{i} \sqrt{ } c_{i}$. Often the $c_{i}$ and sometimes the $S_{i}^{2}$ are treated as equal among strata; then the $U_{i}=W_{i} S_{i} / \Sigma W_{i} S_{i}$ or $U_{i}=W_{i}$. To these parameters the earlier results on optima and proxima can be applied.

For a fixed $m=\sum m_{i}=\sum m_{i}^{*}$, disregarding differences in the $c_{i}$, the loss function (2.7) with sample weights becomes (also shown in Cochran, 1963, 5A.1), with $d_{i}=\left(m_{i}^{*}-m_{i}\right) / m_{i}$ :

$$
L=\frac{V_{\text {actual }}}{V_{\min }}-1=\Sigma \frac{m_{i}}{m} \frac{\left(m_{i}^{*}-m_{i}\right)^{2}}{m_{i}^{2}}=\frac{1}{m} \Sigma \frac{\left(m_{i}^{*}-m_{i}\right)^{2}}{m_{i}}=(1 / m) \Sigma m_{i} d_{i}^{2} .
$$

\subsection{Two Components; Subsampling Non-responses}

For only two components (e.g. two strata or two stages) we can obtain several convenient applications. Optimal numbers of units stand in the ratio

$$
\frac{m_{2}^{*}}{m_{1}^{*}}=\frac{V_{2}}{V_{1}} \sqrt{ }\left(\frac{c_{1}}{c_{2}}\right)
$$

Optimal allocations result in

$$
\text { either } \quad V_{\min }^{2}=\left(V_{1} \sqrt{ } c_{1}+V_{2} \sqrt{ } c_{2}\right)^{2} / C_{f} \text { or } C_{\min }=\left(V_{1} \sqrt{ } c_{1}+V_{2} \sqrt{ } c_{2}\right)^{2} / V_{f} \text {. }
$$

Now let $U=V_{1} \sqrt{ } c_{1} /\left(V_{1} \sqrt{ } c_{1}+V_{2} \sqrt{ } c_{2}\right)$ and $(1-U)$ denote the two population weights; and let $K=k_{2} / k_{1}$ denote the relative departure from optimal numbers, so that $m_{1} / m_{2}=K m_{1}^{*} / m_{2}^{*}$. Choosing the order of components is immaterial for expressing the loss $L$ (from (2.3)), due to departure from optimal, as

$$
1+L=(U K+1-U)(U / K+1-U)=1+U(1-U)\left\{(K-1)^{2} / K\right\} .
$$

For computing the weights we may use any of the relationships

$$
\frac{U}{1-U}=\frac{V_{1} \sqrt{ } c_{1}}{V_{2} \sqrt{c_{2}}}=\frac{m_{1}^{*} c_{1}}{m_{2}^{*} c_{2}}=K \frac{m_{1} c_{1}}{m_{2} c_{2}}=r ; \text { then } U=r /(1+r) \text {. }
$$

The loss is greatest with $U=0.5$ for fixed $K$. It is modest for $0.5<k<2$, but rises sharply for extreme values of $K$ (see Table 1). For example, for a stratified sample we may often assume $S_{2} / \sqrt{ } c_{2}=S_{1} / \sqrt{ } c_{1}$, and optimal allocation would be $m_{2}^{*} / m_{1}^{*}=\left(1-W_{1}\right) / W_{1}$. But if we actually have sample numbers in the ratio $m_{2} / m_{1}=4\left(1-W_{1}\right) / W_{1}$, the departure $K=4$ results in the loss $(9 / 4) W_{1}\left(1-W_{1}\right)$. See column $K=4$ of Table 1 ; also example 2(a) in Section 2.

A ready application is to subsampling of non-responses. Suppose that $m$ questionnaires are mailed out for a total cost of $c_{0} m$; that $R m$ respond to several mailings for a further cost of $c_{r} R m$; and that a fraction $1 / k$ of the $(1-R) m$ non-responses are interviewed for a cost of $c_{n}(1-R) m / k$.

Thus,

$$
\operatorname{cost}(\bar{y})=\left(c_{0} / R+c_{r}\right) R m+c_{n}(1-R) m / k .
$$


Assume either that the $m$ were selected with srs or that design effects are included. Denote with $S_{r}^{2}$ and $S_{n}^{2}$ the element variances of response and non-response. The variance may be written, without the constant factor for finite population, approximately as

$$
\operatorname{var}(\bar{y})=\frac{R^{2} S_{r}^{2}}{R m}+\frac{(1-R)^{2} S_{n}^{2}}{(1-R) m / k}
$$

From (7.2) we get

$$
\text { optimal } k^{*}=\frac{S_{r}}{S_{n}} \sqrt{ }\left(\frac{c_{n}}{c_{0} / R+c_{r}}\right) \text {. }
$$

\subsection{Two-stage and Multistage Selection}

In two-stage random selection without replacement from equal clusters we have

$$
\operatorname{var}(\bar{y})=\left(1-\frac{a}{A}\right) \frac{S_{a}^{2}}{a}+\left(1-\frac{b}{B}\right) \frac{S_{b}^{2}}{a b}=\frac{\left(S_{a}^{2}-S_{b}^{2} / B\right)}{a}+\frac{S_{b}^{2}}{a b}-\frac{S_{a}^{2}}{A},
$$

where the last term denotes the constant $V_{0}$; and $\operatorname{cost}(\tilde{y})=c_{a} a+c_{b} a b+C_{0}$. Using (7.2) the ratio $m_{2}^{*} / m_{1}^{*}=a b / a$ yields

$$
\text { optimal } b^{*}=\frac{S_{b}}{S_{u}} \sqrt{ }\left(\frac{c_{a}}{c_{b}}\right),
$$

where $S_{u}^{2}=S_{a}^{2}-S_{b}^{2} / B$ yields a shorter form that is meaningful: the cluster variance additional to average random variance of its $B$ elements. $S_{u}^{2} \leqslant 0$ would cause a mathematical dilemma because we have assumed positive components, but in practice it leads to taking all $B$ elements, selecting complete clusters. This design $b=B$ also serves when (7.10) yields $b^{*}>B$; and it may be a practical solution even when $b^{*}$ is a large fraction of $B$. In some situations one could search for a new definition of larger clusters. These departures from optimal $b *$ interfere with attaining the optimal values of $V_{\min }$ and $C_{\min }$ (see Section 5).

Extensions to three (or more) stages are not difficult:

$$
\begin{aligned}
\operatorname{var}(\bar{y}) & =\frac{\left(S_{a}^{2}-S_{b}^{2} / B\right)}{a}+\frac{\left(S_{b}^{2}-S_{c}^{2} / C\right)}{a b}+\frac{S_{c}^{2}}{a b c}-\frac{S_{a}^{2}}{A} \\
& =\frac{S_{u}^{2}}{a}+\frac{S_{w}^{2}}{a b}+\frac{S_{c}^{2}}{a b c}-\frac{S_{a}^{2}}{A} .
\end{aligned}
$$

\subsection{Two-phase Sampling}

Suppose a large sample of size $n_{L}$ is selected (with srs) from $N$ in the first phase, and a sample of size $n$ in the second phase. The cost function may be written as

$$
\operatorname{cost}(\tilde{y})=C_{0}+c n+c_{L} n_{L},
$$

where $c_{L}$ and $n_{L}$ denote unit cost and numbers for the large first phase sample, and $c$ and $n$ for the smaller second phase.

When used for proportionate stratification, $n=\sum n_{h}$, and the variance is to a good approximation

$$
\operatorname{var}(\bar{y})=\sum W_{h}^{2} \frac{S_{h}^{2}}{n_{h}}+\frac{1}{n_{L}} \sum W_{h}\left(\bar{Y}_{h}-\bar{Y}\right)^{2}-\frac{\sum W_{h}^{2} S_{h}^{2}}{N_{h}}-\frac{\sum W_{h}\left(\bar{Y}_{h}-\bar{Y}\right)^{2}}{N} .
$$

When fixed $n=\sum n_{h}$ is allocated optimally according to $n_{h}^{*} / n=W_{h} S_{h} / \Sigma W_{h} S_{h}, V_{\min }$ becomes

$$
\operatorname{var}(\bar{y})-V_{0}=\frac{\left(\sum W_{h} S_{h}\right)^{2}}{n}+\frac{1}{n_{L}} \sum W_{h}\left(\bar{Y}_{h}-\bar{Y}\right)^{2}
$$


Allocation of (7.2) and (7.3) between the two cost components of $C=c n+c_{L} n_{L}$ yields

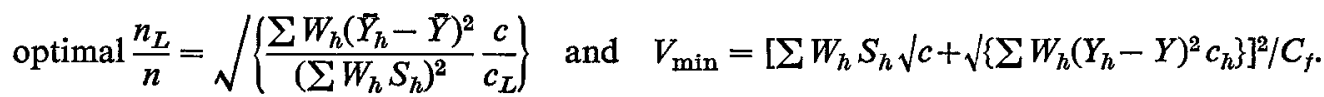

If the cost factors $c_{h}$ vary among the $H$ strata, the optimal $n_{h}^{*}$ should be made proportional to $W_{h} S_{h} / \sqrt{ } c_{h}$. We may solve directly for the $(H+1)$ unknowns $\left(n_{h}\right.$ and $\left.n_{L}\right)$ by applying (5.2) and (5.3) to $C=\sum n_{h} c_{h}+c_{L} n_{L}$, and to (7.13). The first term becomes $\Sigma W_{h} S_{h} \sqrt{ } c_{h}$ in $V_{\min }$.

In two-phase sampling for regression estimation, the variance may be expressed to a good approximation as

$$
V_{(\bar{y})}^{2}=\frac{S^{2}\left(1-R_{y x}^{2}\right)}{n}+\frac{R_{y x}^{2} S^{2}}{n_{L}} .
$$

Allocation of the two cost components yields

$$
\text { optimal } \frac{n_{L}}{n}=\frac{R_{y x}}{\sqrt{1-R_{y x}^{2}}} \frac{\sqrt{ } c}{\sqrt{c_{L}}} \text { and } V_{\min }=S^{2}\left\{\sqrt{ }\left(1-R_{y x}^{2}\right) \sqrt{ } c+R_{y x} \sqrt{ } c_{L}\right\}^{2} / C_{f} .
$$

\subsection{Weights in Estimation}

Our emphasis has been in the allocation of units $m_{i}$ in selection, but the method can also be applied to the allocation of weights $w_{i}$ in linear estimation. Let $y_{i}(i=1,2, \ldots, n)$ be independent estimates of $\bar{Y}$, with $\operatorname{var}\left(y_{i}\right)=\sigma_{i}^{2}$ (with $0<\sigma_{i}<\infty$ ). Suppose one estimates $\bar{Y}$ with a weighted mean $\bar{y}=\Sigma w_{i} y_{i}$, with the constraint $\Sigma w_{i}=1$. How to choose the $w_{i}$ to minimize the variance of $\Sigma w_{i} y_{i}$ ? We denote $\operatorname{var}\left(\Sigma w_{i} y_{i}\right)=\Sigma w_{i}^{2} \sigma_{i}^{2}=V$, and corresponding to $V C$ we form the product

$$
V W=\left(\sum w_{i}^{2} \sigma_{i}^{2}\right)\left(\sum w_{i}\right)=\left(\sum w_{i} \sigma_{i} \sqrt{ } w_{i}\right)^{2}+\sum_{i<j}\left(w_{i} \sigma_{i} \sqrt{ } w_{j}-w_{j} \sigma_{j} \sqrt{ } w_{i}\right)^{2} .
$$

The optimal values $w_{i}^{*}$ are reached when $\sqrt{ } w_{i}^{*} \sigma_{i}=F$, thus

$$
w_{i}^{*}=F^{2} / \sigma_{i}^{2} \text { and } \sum w_{i}^{*}=F^{2} \sum 1 / \sigma_{i}^{2}=1 .
$$

Then

$$
V_{\min }=\Sigma w_{i}^{*} F^{2}=F^{2}=\left(\Sigma 1 / \sigma_{i}^{2}\right)^{-1} \quad \text { and } \quad w_{i}^{*}=\frac{1 / \sigma_{i}^{2}}{\sum 1 / \sigma_{i}^{2}}
$$

The relative loss $L$ may be found from

$$
1+L=V / V_{\min }=\left(\Sigma 1 / \sigma_{i}^{2}\right)\left(\sum w_{i}^{2} \sigma_{i}^{2}\right)=\Sigma \frac{w_{i}^{2}}{w_{i}^{*}}
$$

Thus

$$
L=\sum \frac{\left(w_{i}-w_{i}^{*}\right)^{2}}{w_{i}^{*}}=\Sigma w_{i}^{*}\left(\frac{w_{i}-w_{i}^{*}}{w_{i}^{*}}\right)^{2}=\Sigma w_{i}^{*}\left(k_{i}^{\prime}-1\right)^{2},
$$

with $\Sigma w_{i}^{*} k_{i}^{\prime}=1$, since $\Sigma w_{i}^{*}=\Sigma w_{i}=1$. The loss appears as the relvariance of the relative departures $k_{i}^{\prime}$ (from mean of 1 ) with weights $w_{i}^{*}$-see applications to standardization by Kalton (1968).

For equal variances, $\sigma_{i}^{2}=\sigma^{2}$, the optimal values are also equal, $w_{i}^{*}=1 / n$, and $V_{\min }=\sigma^{2} / n$, and the loss function becomes $1+L=\left(1+C_{\vec{k}}^{2}\right)$, where $C_{k}^{2}$ is the relvariance among the relative departures. When there are only a few values of $k_{i}$ with relative frequencies $W_{i}$, use $(1+L)=\left(\Sigma W_{i} k_{i}^{2}\right) /\left(\Sigma W_{i} k_{i}\right)^{2}$. 
The loss due to unequal weights, $C_{k}^{2}$, represents the discrepancy between them. Therefore, when random replication is used to obtain integral weights (machine cards) from fractions $k+W$, the weighting should be confined to successive integers; with $k$ applied to $(1-W)$ and $(k+1)$ to $W$ of the elements, we have $(1-W) k+W(k+1)=k+W$ cards. The variance is increased by

$$
1+L=\frac{(1-W) k^{2}+W(k+1)^{2}}{(k+W)^{2}}=1+\frac{W(1-W)}{(k+W)^{2}} .
$$

Duplicating for non-response is common, with $k=1$. The loss then is

$$
L=W(1-W) /(1+W)^{2}
$$

its maximum is $0 \cdot 125$, when $W=\frac{1}{3}$. The case of $k=0$ would refer to eliminating $(1-W)$, leading to a loss of $L=(1-W) / W$. Note that eliminating a small fraction $(1-W)$ surprisingly appears only a little worse than duplicating a similarly small fraction $W$. For example, eliminating 0.05 results in $L=0.056$; duplicating 0.05 results in $L=0.046$. Hence to equalize several groups with $k_{i}$ that differ only slightly, instead of duplicating up to the highest response, one may reduce $L$ by eliminating from the groups with the highest response.

\subsection{Allocation Conflict between Totals and Independent Domains}

Serious conflict often exists between reducing the variance for the combined mean $\Sigma W_{i} \bar{y}_{i}$, and equal precision desired for the means $\bar{y}_{i}$ of $H$ independent domains that differ greatly in relative sizes $W_{i}\left(\sum W_{i}=1\right)$. The domains may be the regions or provinces of a country, etc. This common example of multipurpose allocation deserves special attention.

The combined mean variance $V_{c}=\sum W_{i}^{2} S_{i}^{2} / m_{i}$ is minimal when the optimal $m_{c i}^{*} \propto W_{i} S_{i} / \sqrt{ } c_{i}$. However, $m_{d i}^{* *} \propto S_{i} / \sqrt{ } c_{i}$ are optimal for obtaining equal precision for each of the $H$ domain means; also to obtain equal precision for the $H(H-1) / 2$ possible comparisons of domain means. Thus we can denote an average domain variance $V_{d}=\left(\Sigma S_{i}^{2} / m_{i}\right) / H^{2}$ for the variance of $\sum \bar{y}_{i} / H$. The conflict between the purposes is represented in the above two optimal values for $m_{i}^{*}$ by the presence of the weights $W_{i}$ for the combined mean, and their absence for the domain means. Thus the loss function (2.3) for the combined mean, due to allocations $m_{i} \propto S_{i} / \sqrt{ } c_{i}$, has the departures $k_{c i}=m_{i}^{*} / m_{i}=W_{i}$, and the weights $U_{c i} \propto W_{i} S_{i} \sqrt{ } c_{i}$. The loss function for the average domain means, due to allocations $m_{i} \propto W_{i} S_{i} \sqrt{ } c_{i}$, has the departures $k_{d i}=1 / W_{i}$ and the weights $U_{d i} \propto S_{i} \sqrt{ } c_{i}$.

To see clearly the effects of variation in the domain sizes $W_{i}$, we make some simplifying assumptions that are often approximated in practical situations. Assume that the $S_{i}^{2}$ incorporate the effects of complex designs, and that they are constant across domains, as are the $c_{i}$. Further, suppose that $m_{i}^{*} \leqslant M_{i}$ in all domains. We shall also neglect effects of the constants $V_{0}$ and $C_{0}$ on the loss functions.

Under these conditions we may omit, for brevity, the constants $S^{2}$ and $c$ from the formulae, and we allocate the total sample size $m=\sum m_{i}$ among the domains. For $\sum W_{i} \bar{y}_{i}$ the optimal $m_{i}^{*}=m W_{i}$, with departures $k_{c i}=m_{i}^{*} / m_{i}=m W_{i} / m_{i}$ and weights $U_{c i}=W_{i}$, the loss function $1+L_{c}=m \sum W_{i}^{2} / m_{i}$ is minimal at $m V_{c \min }=1$. For $\sum \bar{y}_{i} / H$ the optimal $m_{d i}^{*}=m / H$, weights $U_{d i}=1 / H$, with departures $k_{d i}=m / H m_{i}$ and the loss function $1+L_{d}=m H^{-2} \sum 1 / m_{i}$ is minimal at $m V_{d \min }=1$ also.

In Table 4 for loss functions $\left(1+L_{c}\right)$ the minimal value 1 appears with $m_{i} \propto W_{i}$ for $\sum W_{i} \bar{y}_{i}$, and with $m_{i} \propto 1 / H$ for $\Sigma \bar{y}_{i} / H$. The other allocations produce relative losses $(L>0)$ that increase with diversity among the relative sizes $W_{i}$; and $C_{w}^{2}$ denotes their relative variance, $H^{2} \operatorname{var}\left(W_{i}\right)$.

Jointly for the two purposes, we can find optimal allocation and the loss function with (6.3). For any allocation $m_{i}$, the joint function is

$$
1+L_{j}\left(m_{i}\right)=I_{c} m \sum W_{i}^{2} / m_{i}+I_{d} m H^{-2} \sum 1 / m_{i}=m \sum\left(I_{c} W_{i}^{2}+I_{d} H^{-2}\right) / m_{i}=m \sum_{i} t_{i}^{2} / m_{i},
$$


where

$$
t_{i}=\sqrt{ }\left(I_{c} W_{i}^{2}+I_{d} H^{-2}\right)=\sqrt{ }\left(I_{c} D_{i}^{2}+I_{d}\right) / H=\sqrt{ }\left(I_{c} N_{i}^{2}+I_{d} \bar{N}^{2}\right) / N
$$

Here $0<I_{c}<1$ is the relative importance for the combined mean variance and $I_{d}=1-I_{c}$ for the mean domain variance. We may find it convenient to use $D_{i}=H W_{i}$ with mean $\bar{D}=1$, or $N_{i}=N W_{i}$ when these denote domain sizes and $N=\sum N_{i}=H \bar{N}$.

We can find the joint optimal allocations $m_{i}^{* *}=m t_{i} / \sum t_{i} \operatorname{simply}$ with (5.3), but also as an illustration of (6.8).

\section{TABLE 4}

Conflict of Combined Mean $\left(\sum W_{i} \bar{y}_{i}\right)$ and Average Domain Mean $\left(\sum \bar{y}_{i} / H\right)$ ( $S_{i}^{2}$ and $c_{i}$ are assumed constant and omitted)

Loss function $(1+L)$ for the combined mean, for the average domain mean, and for $a$ weighted joint function

$$
\text { Note } t_{i}=\sqrt{ }\left(I_{c} W_{i}^{2}+I_{d} H^{-2}\right)=\sqrt{ }\left(I_{c} D_{i}^{2}+I_{d}\right) / H
$$

\begin{tabular}{lccc}
\hline & \multicolumn{3}{c}{ Loss functions $(1+L)$ for } \\
\cline { 2 - 4 }$(1+L)=m V^{2}$ & $\begin{array}{c}\sum W_{i} \bar{y}_{i} \\
m \sum W_{i}^{2} / m_{i}\end{array}$ & $\begin{array}{c}\sum_{y_{i}} / H \\
m H^{-2} \sum 1 / m_{i}\end{array}$ & $\begin{array}{c}I_{c} \sum W_{i} \bar{y}_{i}+I_{d} \sum \bar{y}_{i} / H \\
m \sum t_{i}^{2} / m_{i}\end{array}$ \\
\hline $\begin{array}{l}\text { Allocation of } m_{i} \\
m W_{i}\end{array}$ & 1 & $H^{-2} \sum 1 / W_{i}$ & $I_{c}+I_{d} H^{-2} \sum 1 / W_{i}$ \\
$m / H$ & $H \sum W_{i}^{2}=1+C_{b o}^{2}$ & 1 & $I_{c} H \sum W_{i}^{2}+I_{d} H^{-1}$ \\
$m t_{i} / \sum t_{i}$ & $\left(\sum W_{i}^{2} / t_{i}\right)\left(\sum t_{i}\right)$ & $H^{-2}\left(\sum 1 / t_{i}\right)\left(\sum t_{i}\right)$ & $\left(\sum t_{i}\right)^{2}$ \\
\hline
\end{tabular}

TABLE 5

Loss functions $(1+L)$ for two populations

(A)

$(1+L)$ for $W_{1} / W_{2}=4$
(B)

\begin{tabular}{|c|c|c|c|c|c|c|c|}
\hline \multirow[b]{2}{*}{ Allocations $m_{i}$} & \multirow[b]{2}{*}{$\sum W_{i} \bar{y}_{i}$} & \multirow[b]{2}{*}{$\sum \bar{y}_{i} / 2$} & \multirow[b]{2}{*}{ Joint } & \multirow[b]{2}{*}{$\sum W_{i} \bar{y}_{i}$} & \multirow[b]{2}{*}{$\sum \bar{y}_{i} / 133$} & \multicolumn{2}{|c|}{ Joint with weights } \\
\hline & & & & & & $1: 1$ & $I_{c} / I_{d}: 1$ \\
\hline $\begin{array}{l}m W_{i} \\
m / H \\
\propto \sqrt{ } W_{i} \\
\propto \sqrt{ }\left(W_{i}^{2}+H^{-2}\right) \\
\propto \sqrt{\left(0 \cdot 5 W_{i}^{2}+H^{-2}\right)} \\
\propto \sqrt{ }\left(2 W_{i}^{2}+H^{-2}\right) \\
\propto \sqrt{ }\left(4 W_{i}^{2}+H^{-2}\right)\end{array}$ & $\begin{array}{l}1 \\
1 \cdot 36 \\
1 \cdot 08 \\
1 \cdot 116\end{array}$ & $\begin{array}{l}1 \cdot 56 \\
1 \\
1 \cdot 125 \\
1 \cdot 080\end{array}$ & $\begin{array}{l}1 \cdot 28 \\
1 \cdot 18 \\
1 \cdot 102 \\
1 \cdot 098\end{array}$ & $\begin{array}{l}1 \\
3 \cdot 34 \\
1 \cdot 35 \\
1 \cdot 31 \\
1 \cdot 47 \\
1 \cdot 20 \\
1 \cdot 12\end{array}$ & $\begin{array}{l}6.86 \\
1 \\
1.54 \\
1.28 \\
1 \cdot 17 \\
1.44 \\
1.66\end{array}$ & $\begin{array}{l}3.93 \\
2.17 \\
1.44 \\
1.295 \\
(1.32) \\
(1.32) \\
(1.39)\end{array}$ & $\begin{array}{l}1 \cdot 27 \\
1 \cdot 28 \\
1 \cdot 23\end{array}$ \\
\hline
\end{tabular}

$(1+L)$ for 133 countries: 0.2 to $100 \mathrm{~mm}$

In (A) there are two strata and domains $\left(W_{1}=0.8\right.$ and $\left.W_{2}=0.2\right)$; note that the allocation $m_{i}=\sqrt{ } W_{i}$ does almost as well for the joint loss as the optimal.

In (B) we have the populations of 133 countries, ranging in size from 0.2 to over 100 millions, a range of 500 in relative sizes. From this problem of allocation (for the World Fertility Survey) we omitted, for practical reasons, the four largest countries and a few under 0.2 millions. Their inclusion would raise the variance of relative sizes, $W_{i}$, from 2.5 to 12 , and would make the results more dramatic. Note that the $\sqrt{ } W_{i}$ allocation reduces losses quite well. Some compromise is better than none. But the optimal allocation, $\sqrt{ }\left(W_{i}^{2}+H^{-2}\right)$, is considerably better. Different values of $I_{c} / I_{d}(=1 / 2,2 / 1$ and 4/1) increase slightly the variance of the joint loss function with $(1: 1)$ weights; but they remain steady for joint loss functions with their own weights $I_{c} / I_{d}: 1$. 
The multipurpose allocation $m_{i}^{* *}$ can also be shown (5.2) to produce the multipurpose minimal variance

$$
V_{\min }=\left(\Sigma t_{i}\right)^{2} / m
$$

When we use the multipurpose optimal $m_{i}^{* *} \propto t_{i}$ we can determine the loss functions $(1+L)$ incurred for the variances of $\sum W_{i} \bar{y}_{i}$ and $\sum \bar{y}_{h} / H$; we use (6.1) or (6.2) with $k_{c i} \propto V_{c i} / V_{i}^{\prime} \propto W_{i} / t_{i}$ and $k_{d i} \propto V_{d i} / V_{i}^{\prime} \propto 1 / H t_{i}$ respectively. These $(1+L)$ are shown on the bottom row of Table 4 . The last column shows the effects of the three different allocations on the joint multipurpose loss function $1+L_{j}\left(m_{i}\right)$.

Two numerical problems illustrate the method in Table 5. In (A), for two domains having sizes $W_{1} / W_{2}=4: 1$, are shown the loss functions for three purposes-total, domain and joint -under diverse allocations. In (B) the method is applied to the 133 countries of the world, omitting the four largest, over 200 millions, and a few smallest, under 0.2 millions. Including them would be more dramatic but less realistic.

\section{ACKNOWLEDGEMENTS}

The work was supported by Grants GS-777 and GS-3191X from the National Science Foundation. I received help from W. G. Cochran, W. H. DuMouchel and from colleagues in 1969 and 1972 in the Statistics Department of the London School of Economics, especially D. R. Brillinger and G. Kalton. The referee was very helpful.

\section{REFERENCES}

Cochran, W. G. (1963). Sampling Techniques, 2nd ed. New York: Wiley.

Dalenius, T. (1957). Sampling in Sweden. Stockholm: Almquist and Wicksell.

Evans, W. D. (1951). On stratification and optimum allocation. J. Amer. Statist. Ass., 30, 219-229.

Hansen, M. H., Hurwitz, W. N. and Madow, W. G. (1953). Sample Survey Methods and Theory, Vols 1 and 2. New York: Wiley.

HARTLEY, H. O. (1965). Multiple purpose optimum allocation in stratified sampling. Proc. Soc. Statist. Sect. Amer. Statist. Ass., 258-261.

Huddleston, H. F., Claypool, P. L. and Hocking, R. R. (1970). Optimal sample allocation to strata using convex programming. Appl. Statist., 19, 273-278.

Kalton, G. (1968). Standardization: a technique to control for extraneous variables. Appl. Statist., 17, 118-136.

KisH, L. (1961). Efficient allocation of a multipurpose sample. Econometrica, 29, 363-385.

- (1965). Survey Sampling. (Especially Sections 8.5 and 11.7.) New York: Wiley. (1969). Design and estimation for subclasses, comparisons and analytical statistics. Chapter 21 in New Developments in Survey Sampling (N. L. Johnson and H. Smith, eds). New York: Wiley. Kish, L. and Frankel, M. R. (1974). Inference from complex samples (with Discussion). J. R. Statist. Soc. B,
36, 1-37.

KORAN, A. R. and KHAN, S. (1967). Optimum allocation in multivariate surveys; an analytical solution. J. R. Statist. Soc. B, 115-125.

SrIRANTAN, K. S. (1963). A problem in optimum allocation. Operat. Res., 11, 265-273.

STUART, A. (1954). A simple presentation of optimum sample results. J. R. Statist. Soc. B, 16, 239-241.

Sukmatme, P. V. and Sukmatme, B. V. (1970). Sampling Theory of Surveys with Applications, 2nd ed. Ames: Iowa State University Press.

TukeY, J. W. (1948). Approximate weights. J. Amer. Math. Soc., 19, 91-92.

YATES, F. (1960). Sampling Methods for Censuses and Surveys, London: Griffin. 